\title{
Lipid Nanoparticle Encapsulated mRNAs Encoding Human IL-12A/IL-12B MEDI-1191
}

National Cancer Institute

\section{Source}

National Cancer Institute. Lipid Nanoparticle Encapsulated mRNAs Encoding Human IL12A/IL-12B MEDI-1191. NCI Thesaurus. Code C160867.

A formulation consisting of lipid nanoparticle encapsulated messenger RNA (mRNA) encoding human interleukin-12 subunit beta (IL-12B; IL-12 subunit p40) and interleukin12 subunit alpha (IL-12A; IL-12 subunit p35) with potential immunomodulatory and antineoplastic activities. Although the exact mechanism of action has not been completely characterized, upon intratumoral injection, the lipid nanoparticle moiety presumably binds to the plasma membrane of nearby cells and releases the IL-12A and IL-12B mRNA into the cell. The mRNA is then translated by the cellular protein translation machinery to produce a single-chain fusion protein of IL-12B and IL-12A subunits, which is secreted into the local tumor microenvironment (TME). Secretion of IL-12 activates the immune system by promoting the secretion of interferon-gamma, activating natural killer cells (NKs), and inducing cytotoxic T-cell responses, which may result in both decreased tumor cell proliferation and enhanced immune-mediated destruction of tumor cells. 